\title{
Annotated List of the Family Lygaeidae Schilling, 1829 (Heteroptera) in Libya
}

\author{
Moftah Soliman El Meghrai \\ Department of Zoology, Faculty of Science, Benghazi University, Benghazi-Libya
}

Received: 5 June 2020/ Accepted: 30 December 2020

Doi: https://doi.org/10.54172/mjsc.v35i4.335

\begin{abstract}
In this study, subfamilies, genera, and species of Lygaeidae are given, accompanied by synonyms, local and general distribution. This study was carried out during the years 2007-2010 in the country of Libya. This study revealed the presence of 111 species in Libya under 52 genera to 8 subfamilies. 31 are recoded from Libya for the first time in the present study.
\end{abstract}

Keywords: Lygaeidae; Heteroptera; Libya; Synonyms; Local \& General Distribution; Seed Bugs.

\section{INTRODUCTION}

The State of Libya is a country in the Maghreb terranean Sea to the north, Egypt to the east, Sudan to the southeast, Chad to the south, the Niger to the southwest, Algeria to the west, and Tunisia to the northwest. The sovereign state consists of three historical regions: Tripolitania (west), Fezzan (East), and Cyrenica (East). With an area of almost 1.8 square kilometers, Libya is the fourth largest country in Africa.

The family Lygaeidae Schilling (Heteroptera) of Libya is still incompletely known. The earliest work on Libyan Lygaeidae is probably that of (Zavattari, 1934; Damiano 1960; Eckerlein \& Wagner, 1969; Linnavuori, 1969)

This family is a large and well-known group of the order Hemiptera-Heteroptera, \& easily recognized by 4-segmented antennae; located below a line drawn through the middle of the eye, 4-segmented rostrum, forewing with 4 or 5 veins in membrane, female ovipositor usually laciniate, spermatheca usually with distinct bulb and flange. They are often referred to as Seed bugs. region in North Africa, bordered by the Medi-

\section{MATERIALS AND METHODS}

Lygaeid specimens were either hand-collected or by using a sweeping net or sheet-screen while resting on the host plants. Collected specimens were killed by ethyl alcohol, Mounted, and stored. The taxonomic study of the family Pentatomdae was carried out in two steps:-

1-examining the specimens collected during the present work as well as the specimens kept in the main Egyptian reference collections.

2-Identification of specimens was made by the authors based on the relevant literature such as Stichel (1960, 1961), Baranowski, (1990), Harrington (1980), Malipatil (1978), Ashlock (1967), Slater (1985, 1992), Goss (1965), Gross \& Scudder (1963), Scudder (1962c).

\section{RESULTS}

\section{SUBFAMILY: Blissinae}

Genus: Geoblissus Hidaka, 1959

Type species: Geoblissus rotundatus Hidaka, 1959.

*Dimorphopterus brachypterus (Rambur, *Corresponding Author: Moftah Soliman El Meghrai elmitsalis@gmail.com, Department of Zoology, Faculty of Science, Benghazi University, Benghazi-Libya 
1839).

Synonym: Pachymerus brachypterus Rambur, 1839.

Distribution in Libya: Jagbob, Misrata.

General Distribution: France, Japan, Algeria, Cyprus, Egypt, Ethiopia, Sudan, Italy, Spain, Tunisia, Turkey, China, Saudi Arabia, Yemen, India, Chad.

Remarks: Dimorphopterus brachypterus (Rambur, 1839) was listed as Stenoblissus curtulus (Dhrn, 1860) by Wagner \& Slater, 1964.

\section{*Geoblissus hirtulus (Burmeister, 1835)}

Synonym: Geoblissus rotundatus Hidka, 1959

Distribution in Libya: Jagbob, Misrata, Benghazi.

General Distribution: Algeria, Cyprus, Egypt, Ethiopia, Sudan, Libya, Spain, Tunisia, Japan, Turkey, Egypt, China, Yemen, Borneo, India, Chad, Ethiopia, and Sudan.

\section{SUBFAMILY: Lygaeinae}

Genus: Apterola Mulsant \& Rey, 1866

Type species: Apterola kuenckeli M \& R, 1866.

\section{*Apterola (Apterola) kuenckeli M\& R, 1866}

Synonym: Apterola gridellii Mancini, 1942 Boll. Soc. ent. Ital. 92-93.

Distribution in Libya: Cyrenica (Mancni, 1942a)

General Distribution: France, Iberian Pen., Italy, Malta, Sardinia, Sicily, Spain, Algeria, Morocco, Tunisia, and Libya.

Remarks: In Libya (as synonym Apterola gridellii Mancini, 1942).

\section{*Apterola (A) k. kuenckeli M\& R, 1866}

Synonym: Apterola gridellii Mancini, 1942 :

Boll. Soc. ent. Ital. 92-93.

Distribution in Libya: El Merj.

General Distribution: Bulgaria, France, Great Britain, Italy, Malta, Macedonia, Spain, Tunisia, Morocco, Libya, Egypt, and Algeria.

Genus: Cosmopleurus Stal, 1872.

Type species: Lygaeus fulvipes Dallas 1852.
Cosmopleurus fulvipes (Dallas, 1852)

Synonym: Lygaeus fulvipes Dallas, 1852. List. Hem. B. M. 2:536

Distribution in Libya: Fezzan: Wadan, Jerma, El Khomos, Jebel Soda, El Kufra, Houn(Mancini, 1936), Gateron, Ghat, Wadi Iseien, Wadi Tanezzuft, Jebel El Wuenat (Mancini, 1935, 1936a, 1940).

General Distribution: Spain, Croatia, Malta, Algeria, Egypt, Libya, Morocco, Tunisia, Iran, Jordan, Saudi Arabia, Syria, Yemen, Pakistan, and Mauritania.

\section{* Cosmopleurus fulvipes (Dallas, 1852)}

Synonym: Lygaeus degeni Distant, 1918: Ann. Mag. Nat. Hist. (9): 2: 257.

Distribution in Libya: Fezzan, Murzak, Kufra, Obary.

General Distribution: Western Africa, Algeria, Saudi Arabia, Cape Verde, Egypt, Morocco, Iran, Sudan, Syria, and Libya.

\section{Genus: Lygaeus Fabricius, 1794}

Type species: Cimex equestris Linnaeus 1758

\section{Lygaeus creticus Lucas, 1853}

Synonym: Lygaeus sexmaculatus Garbiglietti, 1869, Boll. Soc. ent. Ital. 1:41-112.

Distriution in Libya: Derna (Mancini, 1854)

General Distribution: Libya, Greece, Italy, Saudi Arabia, Afghanistan, Turkey, Cyprus, Iran, Syria, Bosnia, Croatia, Macedonia, Jordan, and Lebanon.

\section{Genus: Hormopleurus Horvath, 1884}

Type species: Hormopleurus nysioides Horvath, 1884

\section{Hormopleurus nysioides Horvath, 1884}

Synonym: Lygaeosoma hoggari Bergevin, 1932 Bull. Soc. Hist. nat. Afr. N. 286-287

Distribution in Libya: Fezzan: Tin Caraden, Bir Tahala (Mancini, 1942a).

General Distribution: Syria, Algeria, Libya, Egypt, Morocco, Iraq, Saudi Arabia, Yemen, Ivory Coast, and Sudan.

Genus Horvathiolus Josifov, 1965

Type species Horvathiolus adonis Linn, 1978. 
Horvathiolus fulvescens (Puton, 1874)

Synonym: Melanocoryphus fulvescens Puton, 1874Pet. Nouv. Ent. 6: 112: 452

Distribution in Libya: Al-Bayda, Jebel Akhdar, Messa (Eckerlein \& Wagner, 1969).

General Distribution: Spain, Algeria, Turkey, Italy, Tunisia, and Libya.

\section{Horvathiolus guttatus (Rambur, 1839)}

Lygaeus guttatus Rambur. 1839 Faun. Andalus, pp. $155-156$

Distribution in Libya: Cyrenica (Eckerlien and Wagner, 1969).

General Distribution: France, Portugal, Spain, Algeria, Egypt, Morocco, Tunisia, and Libya.

\section{*Horvathiolus guttatus guttatus (Ram, 1839)}

Synonym: Melanocoryphus persimilis Horvath, 1916: Ann. Mus. Nat. Hung. 14: 466

Distribution in Libya: El Mej, Gubba

General distribution: Albania, Russia, Cyprus, Portugal, Russia, Caucasus, Algeria, Morocco, Turkey, Tunisia, Spain, and Libya.

Remarks: Horvathiolus guttatus guttatus (Ram, 1839) was listed as Melanocoryphus persimilis Horvath by Zavattari (1934), \& Linnavuori (1965), and Eckerlen \& Wagner 1969 as Horvathiolus persimilis Horvath, 1916, listed as synonym by Vidal 1952.

Genus: Melanocoryphus Stal

Type Species Cimex apuanus Ross, 1794

\section{Melanocoryphus albomaculatus (Goeze, 1778)}

Synonym: Cimex albomaculatus Goeze, 1778. Reuter. Rev.d Ent, 4:202

Distribution in Libya: El Merj (Mancini 1942a).

General Distribution: Holland, Turkey, France, Germany, Romania, Russia, Iran, Italy, and Libya.

\section{*Melanocoryphus albomaculatus albomaculatus Goeze, 1778}

Synonym: Cimex apuanus Rossi, 1794

Distribution in Libya: El Merj, Al-Bayda, and Gubba.
General Distribution: England, Brazil, Turkestan, Caucasus, Astrakhan, Albania, Austria, Belgium, Iran, Czechoslovakia, France, Germany, Greece, Holland, Hungary, Iraq, Italy, Poland, Portugal, Romania, Russia, Spain, Switzerland, Yugoslavia, Algeria, Libya, Turkey, Tadzhikistan Bosnia, Bulgaria, Croatia, Slovakia, Slovenia, Ukraine, Morocco, Tunisia, Azerbaijan, Kazakhstan, Armenia, Kirgizia, Turkmenistan, and Uzbekistan.

\section{Genus: Spilostethus Stal, 1868}

Type species: Cimex militaris Faricius 1775.

\section{Spilostethus pandurus Scopoli, 1763}

Synonym: Cimex pandurus (Scopoli, 1763), Ent. Carn. Exhib. Ins. Carniol. N.363:126.

Distribution in Libya: Jagbob, Tobruk, AlBayda, Benghazi, Zuara, Tripoli, El Homs, Sebha, Misrata, Tajora.

General Distribution: Albania, Andorra, Austria, Bosnia, Bulgaria, Croatia, Czech, Iran, Turkey, France, India, Greece, Hungary, Italy, Macedonia, Malta, Moldavia, Portugal, Spain, Syria, Romania, Russia, Slovakia, Slovenia, Switzerland, Ukraine, Algeria, Egypt, Libya, Morocco, Tunisia, Cyprus, Yemen, China, Armenia, Azerbaijan, Afghanistan, Kuwait, Iraq, Jordan, Turkmenistan, Uzbekistan, Australia, and Philipines.

\section{*Spilostethus (pandurus) pandurus (Scopoli, 173)}

Synonym: Cimex civilis Fabricius, 1787: Mant. Ins. II: 298 (O.D.)

Distriution in Libya: Tobruk, Al-Bayda, Benghazi, and Zoura.

General Distribution: Albania, Austria, Bulgaria, Corsica, Cyprus, Czech, France, Germany, Greece, Iran, Hungary, Italy, Portugal, Russia, Sardinia, Spain, Switzerland, Algeria, Egypt, Morocco, Nigeria, Senegal, Sierra, Leone, Sudan, Kenya, Arabia, Iraq, India, Syria, Turkey, and Australia.

Spilostethus (pandurus) militaris (Fabricius, 1775)

Synonym: Lygaeus militaris (Fab, 1775) 
Distribution in Libya: El Khomos, El Bordy, and Jagbob.

General Distribution: Albania, Austria, Belgium, Bulgaria, Corsica, Cyprus, France, Czechoslovakia, Germany, Greece, Italy, Majorca, Malta, Portugal, Romania, Russia, Italy, Spain, Switzerland, Algeria, Egypt, Ethiopia, Morocco, Indonesia, Iran, Mozmbique, Senegal, Sierra Leone, Sudan, Tunisia, Australia, India, Iraq, Syria, Turkey, and Libya.

\section{Spilostethus saxatilis (Scopoli, 1763)}

Synonym: Cimex saxatilis Scopoli, 1763. Ent. Carn. Exhib. Ins.Carniol., 128:371

Distribution in Libya: Khomos (Mancini1936). General Distribution: Albania, Austria, Bulgaria, Croatia, Czech Republic, France, Italy, Germany, Greece, Hungary, Poland, Portugal, Romania, Russia, Serbia, Slovakia, Switzerland, Turkey, Ukraine, Spain, Slovenia, and Libya.

\section{Spilostethus saxatilis lusitanicus (Herrich-}

Schaeffer, 1850)

Synonym: Lygaeus saxatilis (Scop, 1794)

Distribution in Libya: El Khomos.

General Distribution: France, Portugal, Spain, Algeria, Egypt, Morocco, and Libya.

\section{Spilostethus longulus (Dall, 1852)}

Synonym: Lygaeus longulus Dallas, 1852.

Distribution in Libya: Fezzan, Wadan, Jerma, El Khomos, Jebel Soda, El Kufra, Huon, Gateron, Ghat, Wadi Tanezzuft, Wadi, Iseien, Jebel El Wuenat.

General Distribution: Sudan, Eritrea, Ethiopia, Nigeria, Algeria, Egypt, Arabia, Iran, India, Tunisia, Morocco, Afghanistan, Jordan, Syria, Kuwait, Yemen, and Libya.

Genus: Paranysius Horvath, 1895

Type species: Paranysius fraterculus Hor, 1895

\section{Paranysius fallaciosus (Put 1895)}

Synonym: Paranysius fallaciosus libycus Mancini, 1935: Bull. Soc. Ent. Ital. 67: 79-80

Distribution in Libya: Fezzan, Obari, Wadi Iseil (Mancini, 1936, 1942).

General Distribution: Algeria, Libya, Morocco, Tunisia, and Sudan.

Genus: Caenocoris Fieber, 1860

Type species: Lygaeus nerii Germar, 1847

\section{Caenocoris nerii (Germer, 1847)}

Synonym: Lygaeus semirubes Walker, 1872 : Cat. Hem. Het. B. M. 5: 58

Distribution in Libya: Gharyian (Menozzi 1940), Derna (Mancini, 1942), and Jagbob.

General distribution: Albania, Bulgaria, Cyprus, Russia, Italy, Eritrea, Ethiopia, Mozambiue, China, Syria, Algeria, Malta, Tunisia, Morocco, Turkey, India, Pakistan, Jordan, Saudi Arabia, and Libya.

\section{Genus: Horvathiolus Josifov, 1965}

Type species: Horvathiolus amoenulus (Gerstaecker, 1873)

\section{Horvathiolus fulvescens (Puton, 1874)}

Synonym: Melanocoryphus fulvescens Puton, 1874: Pet. Nouv. Ent. 6: 112: 452

Distribution in Libya: Al-Bayda, Jebel Akhder, and Messa.

General Distribution: Italy, Spain, Algeria, Turkey, Tunisia, and Libya.

\section{Horvathiolus guttatus (Rmbur, 1842)}

Synonym: Melanocoryphus persimilis

Horvath, 1916

Distribution in Libya: El Merj.

General Distribution: France, Portugal, Spain, Algeria, Egypt, Morocco, Tunisia, and Libya.

\section{*Horvathiolus guttatus guttatus (Rambur, 1839)}

Synonym: Melanocoryphus atakoricus Bergevin, E. 1932. Bull. Soc. Hist. nat. Afr. Nord. 23: 281-283.

Distribution in Libya: Derna, El Marj, and Gubba. 
Generall Distribution: Albania, Cyprus, Algeria, Spain, Russia, Morocco, Caucasus, Tunisia, and Libya.

Horvathiolus superbus (Polich, 1781)

Synonym: Cimex superbus Pollich, 1781

Distribution in Libya: El Bakour 60Km east of Benghazi (El Meghrab.M.S.2001).

General Distribution: Albania, Andora, Austria, Belgium, Bosnia, Bulgaria, Canary Isles, and Croatia.

\section{Horvathiolus superbus superbus (Pollich,} 1783)

Synonym: Cimex discolor Pollich,

Gmelin.

Carol. Linn. Syst. Nat. 13th Ed.

Distribution in Libya: El Bakour $60 \mathrm{Km}$ east of Benghazi.

General Distribution: Egypt, Syria, Albania, Austria, Belgium, Bulgaria, Czechoslovakia, France, Germany, Greece, Italy, Poland, Portugal, Russia, Spain, Algeria, Libya, Morocco, and Switzerland.

\section{SUBFAMILY: Pachygronthinae}

Genus: Cymophyes Fieber, 1870

Type species: Cymophyes ochroleuca Fieb, 1870.

Cymophyes ochroleuca Fieber, 1870

Synonym: Cymophyes $(C)$ ochroleuca Fieber, 1870: Verh. Zool. Bot. Ges. Wien. 20: 247-8

Distribution in Libya: Cyrenica (de Bergevin, 1930).

General Distribution: Cyprus, Greece, Russia, Egypt, Caucasus, Syria, Turkey, Turkestan, Iran, and Libya.

\section{SUBFAMILY: Rhyparochromiae}

Genus: Gastrodes Westwood, 1840

Type Species: Gastrodes (Orsillodes) longirostris Puton, 1884.

\section{Gastrodes (Orsillodes) longirostris Puton, 1884}

Synonym: Gastrodes (Orsillodes) longirostris Puton, 1884:a pp. 143-4.
Genus: Hyalochilus Fieber, 1861

Type species: Cymus ovatulus Costa, A.1855

\section{Hyalochilus ovatulus (Costa, 1855)}

Synonym: Hyalochilus cordiger Fieber, 1861: Wien: Gerold vi, Eur. Hem. p. 190.

Distribution in Libya: Wadi Belgader (Mancini, 1942a).

General Distribution: Tunisia, Morocco, France, Turkestan, Canary Isles, Greece, Italy, Malta, Spain, Portugal, Russia, Algeria, and Libya.

Genus: Camptocera Jakovlev, 1877

Type species: Rhyparochromus glaberrimus (Walker, 1872).

\section{Camptocera glaberrima (Walker, 1872)}

Synonym: Tropistethus aurantiacus Distant, 1918: Fn. Brit. Ind. Rhynch. 7: 197

Distribution in Libya: Fezzan, Ghat, (Mancini, 1942), El Khomos (Linnavuori, 1965).

General Distribution: Italy, France, Russia, Spain, Algeria, Egypt, Syria, Turkestan, Iran,

Morocco, Caucasus, Turkey, Eritrea, Sudan, India, and Libya.

Genus: Rhyparochromus Hahn, 1826

Type species: Rhyparochromus pini (L, 1758)

Rhyparochromus

(Microtomideus) carbonarius] (Rambur, 1839)

Synonym: Pachymerus (Microtomideus) dasycnemis Reuter, 1885: Rev. d'Ent., Caen. 4: 221, 223.

Distribution in Libya: El Merj (Mancini 1942a) General Distribution: Germany, Italy, Spain, Algeria, Morocco, and Libya.

\section{*Rhyparochromus (Xanthochilus) saturnius Rossi, 1790}

Synonym: Pachymerus rhombeus Fieber, 1837: Beitr. Ges. Natur- Heilwissen.1: 346.

Distribution in Libya: Benghazi.

General Distribution: Bulgaria, Corfu, Corsica, Crimea, France, Germany, Greece, Italy, Malta, Portugal, Russia, Spain, Algeria, Canary Isles., Egypt, Morocco, Tunisia, Caucasus, Syria, and Libya.

(C) 2020 The Author(s). This open access article is distributed under a CC BY-NC 4.0 license. ISSN: online 2617-2186 print 2617-2178 
Genus: Remaudiereana Hoberlandt, 1954

Type species: Remaudiereana tibialis Hoberlandt, L. 1954: Bull. Inst. franc. Afr. Noire. 16: 922-4

\section{Remaudiereana annnulipes (Baerennsprung, 1859)}

Synnym: Remaudiereana africana Hoberlandt, 1954:Bull. Inst. Franc. Afr. Noire 16: 925-6

Distribution in Libya: Benghazi (Mancini, 1942a), El Merj (Linnavuori,1965).

General Distribution: Burma, Ceylon, Egypt, India, Japan, Sudan, France, Portugal, Libya, Algeria, Morocco, Ethiopia, Iran.

\section{Genus Proderus Fieber, 1861}

Type species: Pachymerus suberythropus, Costa, A.:1841.

\section{Proderus amabilis Puton, 1873}

Synonym: Stenocarenus vulsus Jakovlev, 1876:

Trud. Ent. Ross. 9: 223.

Distribution in Libya: Fezzan: Fewat (Mancini 1942a).

General Distribution: Algeria and Libya.

\section{Genus: Acompus Fieber, 1860}

Type species Acompus rufipes (Wolff, 1802)

Acompus rufipes nigrescens Bergevin, 1930

Synonym: Lygaeus rufipes Wolff, 1804: Icon. Cimic. Desc. Illust. 4: 151, IV. Palm, Erlangae, pp. 127-166.

Distribution in Libya: Cyrenica (de Bergev, 1930 \& Zavattari, 1934).

General Distribution: Austria, Belarus, Belgium, Bosnia, Bulgaria, England, Croatia, Russia, Czech Republic, and Libya.

Genus: Aphanus Laporte \& de Castelnau, 1832

Type species: Aphanus rolandri Linn, 1758.

\section{Aphanus aethiops (Douglas \& Scott, 1868)}

Synonym: Pachymerus rolandri variety morio Gradl, 1881:. Ent. Nach. Machr. 7: 308-9

Distribution in Libya: Magrun 90Km West of Benghazi (Mancini, 1942a).

(C) 2020 The Author(s). This open access article is distributed under a CC BY-NC 4.0 license.

ISSN: online 2617-2186 print 2617-2178
General Distribution: Austria, Cyprus, Czechoslovakia, France, Germany, Greece, Italy, Egypt, Iran, Jordan, Syria, Turkey, and Libya.

\section{Genus: Dieuches Dohrn, 1860}

Type species: Dieuches syriacus Dohrn Dohrn, F.A. 1860. Stettin Ent. Zeit. 21:99-109

\section{Dieuches mucronatus (Stal, 1865)}

Synonym: Dieuches pallidulus Distant, 1904:

Distant: Fn. Brit. Ind. Rhynch. 2: 85.

Distriution in Libya: Tripoitania (El Brakat), Fezzan (Ghat, Tin Alcun) (Mancini,1942a)

General Distribution: India, Arabia, Egypt, Cape Verde Is., Nubia Is., Uganda, and Libya.

Genus: Neurocladus Fieber, 1860

Type species: Rhyparochromus brachiidens Dufour, L.1851

\section{Neurocladus brachiidens (Dufour, 1851)}

Synonym: Neurocladus ater Fieber, 1861.

Distriution in Libya: Benghazi (Mancini, 1936), El Abiar (Mancini,1942a).

General Distribution: Bulgaria, Cyprus, France, Greece, Italy, Portugal, Romania, Russia, Spain, Algeria, Canary Isles., Libya, Madeira, Morocco, Tunisia, Caucasus, Iran, Syreia, Turkestan, and Turkey.

\section{Genus: Gonianotus Fieber, 1861}

Type species: Gonianotus marginepunctatus (Wolff, 1802): Icon. Cimic. 4: 150.

\section{Gonianotus marginepunctatus (Wolff, 1804)}

Synonym: Lygaeus marginepunctatus Wolff, 1804: Icon. Cimic. 4: 150.

Distribution in Libya: Tripolitan, ElAzizia

General Distribution: Austria, Belarus, Belgium, Bosnia, Bulgaria, Russia, and the Czech Republic.

\section{SUBFAMILY: ORSILLINAE}

Genus: Camptocoris Putom, 1886

Type species: Camptocoris maculatus Jakovlev 1885.

Camptocoris longicoris (Putom, 1874)

Synonym: Nysius longicoris Putom, 1874. 
Distribution in Libya: Tripolitan (Leptis Magna).

General Distribution: Italy, Turkey, Syria, Palestine, and Libya.

Genus: Orsillus Dallas, 1852

Type species: Orsillus depressus Dallas, W.S., 1952.

\section{Orsillus maculatus (Fieber, 1861)}

Synonym: Orsillus longirostris Mulsant \&

Rey, 1870: Opusc. Ent. 14: 232

Distribution in Libya: Wadi El Cuf (Mancini, 1942a).

General Distribution: Albania, Bosnia, Bugaria, Croatia, Czech Republic, France, Italy, Greece, Macedonia, Russia, Slovenia, Spain, Ukraine, Azerbaijan, Turkey, Cyprus, Georgia, Jordan, Algeria, and Libya.

\section{* Orsillus maculatus maculatus (Fieber, 1861)}

Synonym: Orsillus longirostris Mulsant \& Rey , 1870: Opusc. Ent. 14: 232

Distribution in Libya: Al-Bayda.

General Distribution: Albania, Austria, France, Greece, Italy, Russia, Spain, Algeria and Libya.

\section{Orsillus depressus Dallas, 1852}

Synonym: Heterogaster depressus Mulsant \& Rey, 1852: Ann. Soc. linn. Lyon 1850-52: 112113.

Distribution in Libya: El Merj (Linnavuori, 1965).

General Distribution: Iran, France, Germany, Hungary, Italy, Portugal, Russia, Spain, Iran, Switzerland, Morocco, Tunisia, Turkey, Albania, Austria, Belgium, Bosnia, Bulgaria, Croatia, Czech Republic, England, Greece, Liechtenstein, Luxembourg, Macedonia, Armenia, Romania, Slovakia, Slovenia, Ukraine, Algeria, Azerbaijan, Kazakhstan, Tadzhikistan, Turkmenistan, Uzbekistan Netherlands, and Libya.

\section{* Orsillus maculatus maculatus (Fieber, 1861)}

Synonym: Orsillus longirostris Mulsant \& Rey , 1870: Opusc. Ent. 14: 232

Distribution in Libya: El Mej.

General Distribution: Albania, Austria, France, Greece, Italy, Russia, Spain, Yugoslavia, And Libya.

\section{Genus: Nysius Dallas 1852}

Type species: Lygaeus thmi Wolff, 1804.

\section{Nysius (Nysius) ericae (Schilling, 1829)}

Synonym: Heterogaster ericae Schilling: Beit. Z.Ent, 1-86.

Distribution in Libya: El Khomos (Mancini, 1942a)

General Distribution: Austria, Belgium, Bosnia, Bulgaria, Egland, Russia, Croatia, and Libya.

\section{Nysius ericae ericae (Schilling, 1829)}

Synonym: Nysius albidus Jakovlev, 1867:

Distribution in Libya: Tripoli

General Distribution: Egypt, Senegal, Astrakhan, Turkestan, USA, Mexico, Panama, Puerto Rico, China, Iran, Austria, Belgium, Iraq, Czech Republic, England, France, Germany, Hungary, Poland, Armenia, Portugal, Romania, Russia, Spain, Sweden, Arabia, Switzerland, Yugoslavia, Algeria, Mongolia, Bulgaria, Croatia, Greece, Slovakia, Ukraine, Morocco, Tunisia, Yemen, Turkey, Taiwan, Armenia, and Libya.

\section{Nysius (Macroparius) cymoides (Spinola,} 1837)

Synonym: Arthenesis cymoides Spinola, 1837: Essai, Gen. Ins.Hem:252

Distribution in Libya: Sahabi, Gialo, El Kufa (Zavattari, 1934).

General Distribution: Russia, Turkey, Spain, Sudan, and Libya.

\section{*Nysius senecionis senecionis (Schilling, 1829)}

Synonym: Nysius senecionis aegypticus Priesner \& Alfieri, 1953:Bull.Soc.Fouad.

Ent.37:43.

Distribution in Libya: Ajdabiya (de Bergevin, 1932).

General Distribution: Sudan, Syria, Albania, Austria, Belgium, Bulgaria, Mongolia, France, (C) 2020 The Author(s). This open access article is distributed under a CC BY-NC 4.0 license. ISSN: online 2617-2186 print 2617-2178 
Germany, Greece, Hungary, Italy, Poland, Portugal, Egypt, Armenia, Croatia, Romania, Russia, Spain, Switzerland, Algeria, Yemen, Morocco, China, Turkey, Denmark, England, Netherland, Slovakia, Slovenia, Ukraine, Tunisia, Kazakhstan, Turkmenistan, Uzbekistan, Czech Republic, Saudi Arabia and Libya.

*Nysius immunis (Walker, 1872)

Synonym: Nysius stalianus Horvath, 1890.

Distribution in Libya: El Bieda

General Distribution: France, Greece, Italy, Algeria, Morocco, Turkey, Canary Isles, Croatia, France, Italy, Portugal, Britain, Spain, Switzerland, Algeria, Morocco, Tunisia, Turkey, and Libya.

Remarks: species Nysius (Tropinysius) stalianus (Walker, 1872) was synonymized as species Nysius immunis (Walker, 1872).

\section{SUBFAMILY: Geocorinae}

\section{Genus Geocoris Fallen, 1814}

Type Species Cimex grylloides Linnaeus, 1761,

\section{Geocoris (Eillatus) confalonierii de Bergvin,} (1932)

Synonym: Geocoris massoni Villiers, 1956 : Villiers, A. 1956. Bull. Inst. franc. Afr. N.

Noire 18: 840-841.

Distibution in Libya: El Kufra, Bezema (Mancini, 1940).

General Distribution: Mauritania, Algeria, Egypt, Sudan, and Libya.

\section{Geocoris (Geocoris) acuticeps Signoret, 1881}

Synonym: Geocoris acuticeps falsatus Montandon, 1916: Bull. Soc. Ent. Egypt 4: 50

Distribution in Libya: Fezzan: Murzuk (Mancini, 1942a).

General Distribution: Greece, Malta, Egypt, Iran, Iraq, Saudi Arabia, Uzbekistan, Turkmenistan, Tadzhikistan, Yemen, Sudan, Kuwait, Syria, and Libya.

Geocoris (Geocoris) lineolus (Rambur, 1839) Synonym: Geocoris lineola Ramur, Linnavouri 1978: Acta.Zoo.Fenn.153:60.
Distribution in Libya: Tripolitania: Leptis Magna, Misrata (Mancini, 1936).

General distribution: Europe and Africa.

\section{*Geocoris (Geocoris) lineolus lineolus (Ramb} ur, 1839)

Synonym: Geocoris erythrophthalmus Reuter:.

Ofvers. Finsk: Ofv. Finsk. Vet. Soc. Forh. 22: 9

Distribution in Libya: Benghazi.

General Distribution: Turkey; Corsica, Russia, Caucasus, France, Greece, Italy, Portugal, Spain, Algeria, Canary Isles., Egypt, Tunisia, Morocco, Iran, Syria, Saudi Arabia, Turkey, Iraq, Cyprus, Albania, Bosnia, Bulgaria, Yemen, and Libya.

\section{Geocoris nigriceps Reuter, 1891}

Synonym: Geocoris nigriceps Reuter, 1891.

Distribution in Libya: Fezzan: Sebha, Ain Kirim, Tripolitania: Misrata, El Homos (Mancini, 1936), Kufra (Mancini, 1940).

General Distribution: Egypt, and Libya.

\section{Geocoris nigriceps henoni Puton, 1892}

Synonym: Geocoris nigriceps henoni Puton, 1892.

Distribution in Libya: Fezzan: Sebha, Ain Kirim, Tripolitania: Misrata, El Homos (Mancini, 1936), Kufra (Mancini, 1940).

General Distribution: Palestine, Egypt, and Libya.

\section{*Geocoris (Geocoris) erythrops (Dufour, 1857)}

Remarks: Species Geocoris scutellaris Puton, 1886 was synonymized as species Geocoris erythrops (Dufour, 1857).

Distribution in Libya: El Kufra, El Giof, El Giululad, Bir El Achuan, El Wenat, El Talab, Gialo, Wadi El Ghaza (Mancini,1940).

General Distribution: Algeria, Egypt, Tunisia, Kurdistan, Cape Verde Is, and Libya.

\section{Geocoris (Geocoris) megacephalus (Rossi, 1790)}

Synonym: Ophthalmicus phaeopterus Germar, 1837: Silber. Rev. Ent. 5: 137

Distribution in Libya: Jagbob (de Bergevin, 1930). 
General Distribution: Albania, Austria, Belgium, Bosnia, Bulgaria, Croatia, France, Syria

Germany, Greece, Hungary, Italy, Macedonia, Malta, Netherlands, Poland, Romania, Iraq Russia, Slovenia, Spain, Algeria, Egypt, Iran, Morocco, Tunisia, Afghanistan, Azerbaijan, Uzbekistan, Armenia, Turkey, Cyprus, Jordan, and Libya.

\section{Geocoris (Geocoris) collaris Puton, 1878}

Synonym: Geocoris thoracicus Puton, 1874 : Pet. Nouv. Ent. 6: 452.

Distribution in Libya: Huon (Mancini, 1942, Gialo).

General Distribution: Algeria, Canary Isles, Iraq, Egypt, Morocco, Tunisia, Saudi Arabia, Somalia, Yemen, Ethiopia, Pakistan, and Libya.

*Geocoris (Piocoris) erythrocephalus erythroc ephalus (Lepelitier \& Serville, 1825)

Synonym: Geocoris scutellaris Puton, 1886.

Distriution in Libya: Sebha, El kufra.

General Distribution: Albania, Bulgaria, Corfu, Crimea, Cyprus, Czechoslovakia, France, Germany, Italy, Iraq, Greece, Hungary, Portugal, Romania, Russia, Spain, Algeria, Egypt, Morocco, Astrakhan, Caucasus, Lebanon, Syria, Turkestan, Turkey, and Libya.

Geocoris (Piocoris) luridus (Fieber, 1844). Synonym: Ophthalmicus luridus Fieber, 1844. Distribution in Libya: El Kufra, Sebha, Ain Kirim, El Giof, El Giululad (Mancini, 1942), Jagbob (de Bergevin,1930), Gialo, Augia, (de Bergevin, 1932).

General Distriution: Algeria, Egypt, Tunisia, Sudan, Saudi Arabia, and Libya.

\section{*Geocoris (Piocoris) luridus luridus (Fieber, 1844)}

Synonym: Piocoris obesus Stal, 1872.

Distribution in Libya: El Kufra, Sebha, Gialo. General Distribution: Algeria; Egypt; Iraq, Turkmenstan, Tunisia, Sudan, Arabia, Caucasus, Iran, Syria, Turkestan, Turkey, Morocco, Tunisia, Uzb-ekistan, Azerbaijan,
Armenia, Georgia, Jordan Iran, SaudiArabia, Tadzhikistan, Ethiopia, Yemen, Sudan, Turkmenistan, and Libya.

\section{Genus: Stenophthalmicus Costa, 1875}

Type species: Stenophthalmicus fajoumensis Costa, A., 1875.

\section{Stenophthalmicus fajoumensis Costa, 1875}

Synonym:

Stenophthalmicus mixtus Montandon, $\quad$ 1897:

Rev. d'Ent. 16: 98-99.

Distribution in Libya, Tajura, Zuara, Triploi.

General Distribution: Algeria, Egypt, Tunisia, Sudan, Cyprus, Greece, Italy, Spain, and Libya.

\section{Stenophthalmicus biskrensis Puton, 1887.}

Synonym: Stenophthalmicus biskrensis Puton,

1887: Bull. Soc. Ent. Egypt. 4: 51

Distribution in Libya: Fezzan: Wadi Tanezzuft (Mancini, 1936a).

General Distribution: Algeria, Egupt, Tunisia, Turkestan, Mauritania, Somalia, Niger, Iran, Morocco, Turkmenistan, and Libya.

\section{SUBFAMILY: Henestarinae}

Genus: Henestaris Spinola, 1837

Type species: Henestaris genei Spinola, M.1837: Essai Gen. Hem. pp. 228-230.

\section{* Henestaris laticeps laticeps (Curtis, 1837)}

Synonym: Henestaris curtulus Horvath, 1911:. Bull. Soc. ent. Egypt $>>$ Note: Bull. Soc. Ent. Egypt 2: 106-7.

Remarks: species Henestaris curtulus Horvath, 1911 was synonymized a species Henestaris laticeps laticeps (Curtis, 1837).

Distribution in Libya: El Bordy $(100 \mathrm{Km}$ east Tobruk).

General distribution: France, Algeria, Greece, Spain, Algeria, Egypt, Morocco, Austria, Belgium, Germany, Albania, Bulgaria, England, Croatia, Italy, Portugal, Turkey, Spain, and Libya. 
Genus: Engistus Fieber, 1864

Type species: Engistus brucki Fieber:. 1864. Wien. Ent. Mschr. Ent. Monat. 8: 67-8.

\section{Engistus boops (Dufour, 1857)}

Synonym: Engistus boops (Dufour, 1857): Ann. Ent. Soc. Fr. (3): 5: 70.

Distribution in Libya: Zuara, Tripoli (Linvouri, 1965).

General distribution: Greece, France, Portugal, Italy; Spain, Algeria, Morocco, Tunisia, Canary Isles., Cyprus, Egypt, and Libya.

\section{*Engistus boops boops (Dufour, 1857)}

Synonym: Engistus brucki Fieber, 1864 Fieber: Wien. Ent. Mschr. Monat. 8: 68.

Distribution in Libya: Zuara, Tripoli, Benghazi. General Distribution: Spain, Algeria, Portugal, Greece, France, Italy, Cyprus, Egypt, Morocco, Canary Isles., Tunisia, and Libya.

\section{* Engistus exsanguis exsanguis Stal, 1872}

Distribution in Libya: Jebel El Wenat.

General distribution: France, Astrakhan, Cabo Verde, Turkestan, Morocco, Tunisia, Iran, Spain, Tukey, Russia, Algeria, Egypt, Greece, Ukraine, Syria, Canary Isles, Afghanistan, Kazakhstan, Iraq, Armenia, Iraq, Jordan, Kirgizia, Kuwait, Saudi Arabia, Sudan, Turkmenistan, Uzbekistan, Tadzhikistan, Pakistan, and Libya.

\section{Engistus exsanguis (Stal, 1873)}

Synonym: Engistus exsanguis Stal, C. 1872. Ofvers. Vetensk Akad. Forh, Stockh. $>>$ Note: Ofv. Vet. Akad. Forh. 29: 45.

Distribution in Libya: Jagbob Oasis El Wenat, Murzzuk (Mancini, 1935, 1940, 1942).

General Distribution: Turkestan, Kuwait, Iran, Eremian zone, Sudan, Pakistan, and Libya.

\section{SUBFAMILY: Cyminae}

\section{Genus Cymus Hahn, 1831}

Type species: Lygaeus claviculus Fallen, 1807

\section{Cymus minutus Lindbg, 1939}

Synonym: Cymus minutus Lindbg, 1939:Bull. Soc. Fouad Ent. 22: 14-15.
Distribution in Libya: El Kufra, Benzema, El Giof (Mancini, 1940), Ghat (Mancini, 1942).

General Distribution: Egypt, Syria, Turkey, Saudi Arabia, Algeria, and Libya.

\section{*Cymus gracilicornis Vidal, 1940}

Synonym: Cymus gracilicornis Vidal, 1940:Bull. Soc. Hist. Nat. Afr. Nord 30: 442.

Distribution in Libya: El Kufra.

General Distribution: Canary Isles., Cape Verde Isles, Morocco, Sudan, Albania, Egypt, Tunisia, Turkey, Iraq, Saudi Arabia, Yemen, Iran, Libya.

\section{SUBFAMILY: Heteogastrinae}

Genus: Heterogaaster Schilling, 1829

Type species: Cimex urticae Fabricius, J.C., 1775.

\section{Heterogaster nasuta Horvath, 1895}

Synonym: Heterogaster nasuta Horvath, G. 1895. Rev. Ent., Caen. 14: 157-8

Distribution in Libya: Al-Bayda.

General Distribution: Algeria, Morocco, Tunisia, and Libya.

*Heterogaster cathariae (Geoffroy, 1785)

Synonym: Phygas nepetae Fieber, 1837: Beitr. Ges. Natur.-Heil.-Wiss 1: 348

Distribution in Libya: Jebel Akhder.

General Distribution: Andorra, Austria, Bosnia, Bulgaria, Croatia, Romania, Germany, Italy, Czech Republic, France, Greece, Hungary, Moldavia, Russia, Switzerland, Slovakia, Spain, Ukraine, Yugoslavia, Algeria, Morocco, Azerbaijan, China, Kazakhstan, Armenia, Turkey, Cyprus, Georgia, Iran, Kirgizia, Tadzhikistan, Uzbekistan, Turkmenistan, and Libya.

Genus: Platyplax Fieber, 180

Type species: Heterogaster salviae Schilling, 1829

Platyplax inermis (Rambur, 1839)

Synonym: Heterogaster salviae Schilling, 1829.

Distribution in Libya: El Merj. 
General Distribution: Albania, Bosnia, Hercegovina, Croatia, France, Greece, Macedonia, Italy, Portugal, Spain, Algeria, Canary Isles, Yemen, Ethiopia, Tunsia, Cyprus, Turkey, Morocco, Tunisia, and Libya.

\section{SUBFAMILY: Oxycarninae}

\section{Genus: Oxycarenus Fieber, 1860}

Type species: Stenogaster tardus Hahn (=Oxycarenus lavaterae), 1883.

\section{Oxycarenus hyalinipennis (A. Costa, 1847)} Aphanus hyalinipennis A.Costa, 1843: Att. R. Ist. Sci. Nat. Napol. 7: 184-185.

Distribution in Libya: Cyrenica (1942a).

General Distribution: Bosnia and Herzegovina, Bulgaria, Croatia, France, Greece, Italy, Portugal, Spain, Albania, and Libya.

\section{*Oxycarenus (Oxycarenus) hyalinipennis (Co sta, 1847) \\ Synonym: Oxycarenus castaneus Bergevin, 1932 Bull. Soc. Hist. nat. Afr. N. \\ Distribution in Libya: El Merj.}

General Distribution: Argentina, Bolivia, Brazil Paraguay, Tunisia, Angola, Austria, France, Italy, Greece, Hungary, Italy, Portugal, Russia, Spain, Algeria, Egypt, Morocco, Sudan, Ethiopia, Iraq, Congo, Ethiopia, Kenya, Madagascar, Syria, Mauritania, Mozambique, Rawanda, Sengal, Uganda, Burma, China, India, Iran, and Libya.

\section{Oxycarenus pallens (Herrich-Schaeffer \& G.H.W., 1850)}

Synonym: Stenogaster pallens HerrichSchaeffer, 1850: Wanz. Ins. 9: 214-216.

Distribution in Libya: Jebel Nefousa.

General distribution: Holomediterranean, extending to Middle and Central Asia, India, Sudan, Turkestan, and Libya.

Genus: Bycanistellus Reuter, 1890

Type species: Bycanistes naso Stal, C. 1872: Ofv. Vet. Akad. Forh. 29: 50.
Akad. Forh. 29: 50.

Distribution in Libya: Tobruk, and El Khomos. General Algeria, Egypt, Morocco, Tunisia, and Libya.

Genus: Leptodemus Reuter, 1900

Type species: Leptodemus minutus variety pallidula Reuter, O.M,1900.

\section{Leptodemus bicolor Lindberg, 1924}

Synonym: Cyproplax avenae Lindberg,

Comment. Biol. 10: 7: 68, 69, 167.

Distribution in Libya: Jebel Nefousa, Gharyian. General distribution: Greece, Italy, Egypt, Iran, Greece, Italy, Egypt, Cyprus, Tadzhikistan, Iran, Turkmenistan, Kirgizia, Uzbekistan, Iraq, Libya.

Leptodemus bicolor ventralis Schmidt, 1939 Leptodemus bicolor variety ventralis Bull. Soc. Fouad Ent. 23: 20-21.

Distribution in Libya: Jebel Nefousa, Gharyian. General Distribution: Egypt, and Libya.

\section{Leptodemus minutus (Jakovlev, 1876)}

Synonym: Leptodemus scorteccii Mancini, 1936 Att. Soc. Ital. Sci. Nat. 75: 303-304 Distribution in Libya: El Khomos (Mancini, 1936), Kufra-Benezema, Giaidi, Haufun, kufra1 Tag, Bir El Harraso, El Weinat-Ain Murr, Ain Doua (Mancini, 1940), Gialo (deBergreven, 1932). Benghazi, Gialo (Zavattari, 1934).

General Distribution: Algeria, Turkey, Saudi Arabia, Morocco, Tunisia, Afghanistan, Iran, Egypt, Jordan, Kirgizia, Kuwait, Mongolia, Iraq, Canary Isles, Cyprus, Turkey, Kazakhstan, Saudi Arabia, Azerbaijan, Tadzhikistan, Turkmenistan, Iraq, Syria, Yemen, Uzbekistan, and Libya.

Remarks Leptodemus scorteccii Mancini, 1936.

Synonym: of Leptodemus minutus (Jakovlev, 1876).

*Leptodemus minutus minutus (Jakovlev, 1876).

\section{Bycanistellus naso (Stal, 1872)}

Bycanistellus naso (Stal, 1872): Ofv. Vet. (C) 2020 The Author(s). This open access article is distributed under a CC BY-NC 4.0 license. ISSN: online 2617-2186 print 2617-2178 
Synonym: Leptodemus scorteccii Mancini, 1936: Att. Soc. Ital. Sci. Nat. 75: 303-304

Distribution in Libya: Benghazi, and Gialo.

General Distribution: Cyprus, Russia, Algeria, Egypt, Morocco, Mauritania, Astrakhan, Caucasus, Turkestan, Turkey, and Libya.

\section{Genus: Microplax Fieber, 1860}

Type species: Oxycarenus plagiatus Fieber, F.X., 1860.

\section{Microplax plagiatus (Fieber, 1837)}

Synonym: Microplax plagiatus (Fieber, 1837)

Beitr. Ges. Nat. Heilwiss. p. 342.

Distribution in Libya: Jebel Nefousa, Ghryian, Azizia, Gaser El Garabulli, Misrata (Mancini,1936).

General Distribution: Portugal, Italy, Algeria, Morocco, Tunisia, and Libya.

\section{SUBFAMILY: Aphaninae}

\section{Genus: Megalonotus Fieber, 1860}

Type species: Lygaeus chiragra Fabric 1794. (= Megalonotus chiragrus chiragrus)

\section{Megalonotus puncticollis (Luc, 1849)}

Synonym: Megalonotus praetextatus (HerrichSchäffer, 1850): Wanz. Ins. 4: 12-13.

Distribution in Libya: Khomos (Mancini, 193) General Distribution: Algeria; Canary Isles, Morocco, Caucasus, Tunisia, Syria, Spain, France, Greece, Italy, and Libya.

\section{*Megalonotus (Megalonotus) puncticollis pun} cticollis (Lucas, 1849)

Synonym: Rhyparochromus luctuosus Lucas, 1849: Expl. Alger. Zool. 3: 3: 77.

Distribution in Libya: Gharyian

General distribution: Albania, France, Italy, Greece, Italy, Russia, Spain, Yugoslavia, Algeria, Canary Isles, Morocco, Caucasus, Tunisia, Syria, and Libya.

\section{Genus Aellopus Wolf, 1811}

Type species: Cimex atratus Goeze, 1778.

\section{Aellopus atratus (Gz, 1778)}

Synonym Cimex atratus (Goeze, 1778) Ent.
Beytr. Ritter Linne 2: 268.

Distribution in Libya: Cyrenica (Mancini, 1942a).

General Distribution: Austria, Cyprus, Bulgaria, Belgium, Bosnia, Croatia, Cyprus, Czech Republic, Turkey, and Libya

\section{*Aellopus atratus atratus Goeze, 1778}

Synonym Lygaeus aterrimus Fabricius, 1798:

Supp. Ent. Syst

Distribution in Libya: Benghazi.

General Distribution: Austria, Belgium, Italy, Bulgaria, Cyprus, France, England, The Czech Republic, Germany, Greece, Hungary, Holland, Spain, Portugal, Poland, Romania, Switzerland, Iran, Russia, Algeria, Morocco, Tunisia, Iran, India Syria, Turkestan, Turkey, Astrakhan, Caucasus, and Libya.

\section{Genus: Taphropeltus Stal, 1872}

Type species: Lygaeus hamulatus Thomson, 1870.

\section{Taphropeltus nervosus (Fieber, 1861)}

Synonym: Scolopostethus nervosus Fieber, 186. Wien: Gerold vi, 1: Eur. Hem. p. 189

Distribution in Libya: Wadi-Belgader (Mancini, 1942a).

General Distribution: Croatia, Cyprus, Turkey, Italy, Malta and Libya.

\section{*Taphropeltus nervosus nervosus (Fieber, 1861)}

Synonym: Taphropeltus nervosus nervosus (Fieber, 1861), Wien: Ger vi, E. H. p. 189 Distribution in Libya: El Wueinat.

General Distribution: Portugal, France, Italy, Greece, Spain, Algeria, Morocco, Tunisia, Syria, and Libya.

\section{Genus Scolopostethus Fieber, 1860}

Type species:Tritomacera aphanoides

Fieber, 1860 (= Scolopostethus cognatus).

\section{Scolopostethus decoratus (Hhn, 1831)}

Synonym: Scolopostethus brevis Saunders, 1876: Ent. Mo. Mag. 12: 221

Distribution in Libya: Derna (Mancini, 1942a). 
General Distribution: France, Germany, Holland, Spain, Algeria Albania, Austria, Italy, England, Bulgaria, Denmark, Estonia, Poland, Belgium, Greece, Hungary, Ireland, Czech Republic, and Libya.

Genus: Lethaeus Dall, 1852

Type species: Lethaeus africanus Dallas, 1852 ,

\section{Lehaeus lethierryi (Put, 1869)}

Synonym: Lethaeus lethierryi lethierryi (Puton, 1869) Ann. Soc. Ent. Fr. p. 142

Distribution in Libya: El Bordy, Cyrenica (de Bergevin, 1930, Zavattari, 1934).

General Distribution: Algeria;Arabia;Canary Isles, Cape Verde Isles, Egypt, Mauritania, Iran, Tunisia, Caucasus, Iran, Syria, Jordan, Turkey, and Libya.

\section{Genus: Lasiocoris Fieer, 1860}

Type species: Pachymerus anomalus Kolenati, 1845. (=Lasiocoris anomalus anomalus)

\section{Lasiocoris crassicornis (Luc, 1849)}

Synonym: Lasiocoris antennatus Montandon, 1889: Rev. d'Ent. 8: 288

Distribution in Libya: Cyrenica (Mancini, 1942a).

General Distribution: Greece, Italy, Turkey, Iran, Turkestan, Algeria, and Libya.

\section{Genus: Peritrechus Fieber, 1860}

Type species: Beosus angusticollis Sahlberg, 1848.

\section{Peritrechus meridionalis Put, 1877}

Synonym: Peritrechus meridionalis Put, 1877: Pet. Nouv. Ent. 2: 168: 117.

Distribution in Libya: Cyrenica (Mancini, 1942a).

General Distribution: Algeria, and Libya.

\section{Peritrechus meridionalis fuscatus Ferrari, 1888}

Syn: Peritrechus meridionalis fuscatus Ferrari, 1888:Ann. Mus. Civ. Stor. Nat. Gen. (2): 6: $545,557$.
Distribution in Libya: Cyrenica (Mancini, 1942a).

General Distribution: France, Italy, Algeria, Libya.

Genus: Stygnocoris Douglas \& Scott, 1860

Type species: Lygaeus rusticus Fallen, 1807 (= Stygnocoris rusticus rusticus)

Stygnocoris rusticus (Fallen, 1807)

Synonym: Lygaeus rusticus Fallen, 1807:

Monog. Cim. Suec. p. 70.

Distribution in Libya: El Bordy (de Bergevin, 1930, Zavattari, 1932).

General Distribution: Austria, Belarus, Russia, Belgium, Bosnia, England, Bosnia, Bulgaria, Czech Republic, Croatia, and Libya.

*Stygnocoris rusticus rusticus (Fallen, 1807)

Synonym Stethotropis incana Fieber, 1870 , Verh. Zool. Bot. Ges. Wien. pp. 245-6.

Distribution in Libya: Tobruk.

General Distribution: Austria, Belgium, Spain, Denmark, Italy, England, Estonia, The Czech Republic, Finland, France, Germany, Hungary, Holland, Hungary, Ireland, Holland, Portugal, Poland, Romania, Russia, Sweden, Switzerland, Wales, Caucasus, Astrakhan, Algeria, Morocco, USA, Canada, and Libya.

\section{Genus: Camptocera Jakowleff, 1877}

Type species: Rhyparochromus glaberrimus Walker, 1872 (= Camptocera glaberrima).

\section{Camptocera glaberrima (Walker, 1872)}

Synonym: Tropistethus aurantiacus Distant, 1918Fn. Brit. Ind. Rhynch. 7: 197.

Distribution in Libya: Ghat (Mancini, 1942a), El Khomos (Linnavuori, 1965).

General Distribution: France, Russia, Spain, Algeria, Canary Isles, Egypt, Morocco, Astrakhan, Caucasus, Iran, Israel, Syria, Turkestan, Turkey, Eritrea, Sudan, India, Iran, and Libya.

\section{Genus: Ischnopeza Fieber, 1860}

Type species: Pachymerus hirticornis HerrichSchaeffer, 1850.

\section{Ischnopeza pallipes Put, 11892}

(C) 2020 The Author(s). This open access article is distributed under a CC BY-NC 4.0 license. ISSN: online 2617-2186 print 2617-2178 
Ischnopeza pallipes Put, 11892:Rev. d'Ent. 11: 27.

Distribution in Libya: Merj (Mancini, 1942a). General Distribution: Cyprus, France, Algeria, Egypt, Lebanon, Syria, Turkey, and Libya.

\section{Genus Emblethis Fieber, 1860}

Type species: Lygaeus verbasci Fabricius, 1803 (= Emblethis verbasci verbasci)

\section{Emblethis ciliatus Horvath, 1875}

Synonym: Emblethis ciliatus Horv, 1875.

Distribution in Libya: El Bordy (de Bergevin, 1936, Zavattori, 1934).

General Distribution: Austria, Bulgaria, Crete, The Czech Republic, Germany, Greece, Hungary, Romania, Russia, Egypt, Morocco, Turkey, Iraq, Caucasus, Kurdistan, Siberia, Syria, Algeria, and Libya.

\section{Emblethis verbasci (F, 1803)}

Synonym: Lygaeus verbasci Fabricius, 1803.

Distribution in Libya: Benghazi (Mancini, 1942a), El Bordy (de Bergevin, 1930, Zavattari, 1934).

General Distribution: Turkey, Kazakhstan, Turkestan, France, Iran, Ukraine, Italy, Poland, Spain, and Libya.

*Emblethis verbasci verbasci $(\mathrm{F}, 1803)$

Synonym: Lygaeus pilifrons Zetterstedt, 1819: Kongl. Vet. Akad. Handl. pp. 71-2.

Distribution in Libya: El Merj, Benghazi.

General Distribution: Albania, Austria, Cyprus, Bulgaria, Poland, Belgium, England, Germany, Iran Holland, France, Greece, Hungary, Switzerland, Malta, Spain, Portugal, Czechoslovakia, Syria, Romania, Russia, Sweden, Yugoslavia, Algeria, Afghanistan, Egypt, Morocco, Turkey, Canary Isles, Tunisia, Mongolia, and Libya.

\section{Emblethis angustus Mont, 1890}

Synonym: Emblethis angustus Montandon, 1890

Distribution in Libya: $60 \mathrm{Km}$ east of Nalut.

General Distribution: Bulgaria, Portugal, Canary Isles, Croatia, Italy, Macedonia, and Libya.

(C) 2020 The Author(s). This open access article is distributed under a CC BY-NC 4.0 license.

ISSN: online 2617-2186 print 2617-2178
Emblethis gracilicoris Put, 1883

Synonym: Emblethis oblongus Wagner, 1959.

Distribution in Libya: Jebel Soda, Valle Uosca (Mancini, 1936).

General Distribution: Spain, Algeria, Egypt, Morocco, Arabia, Iran, Kenya, Iran, and Libya.

\section{Genus: Macropternella Slater, 1957}

Type species: Oxycarenus inermis Fieber, 1852.

\section{Macropternella marginalis (Fieb, 1861)}

Synonym: Macropternella marginalis Fieber, 1861.

Distribution in Libya: $20 \mathrm{Km}$ west Azizia.

General Distribution: France, Italy, Portugal, Spain, Morocco, West Africa, and Libya.

\section{Genus: Brachyplax Fieber, 1860}

Type species: Brachyplax albidus Fieber, 1860. (= tenuis tenuis).

\section{Brachyplax tenuis (Mulsant \& Rey, 1852)}

Synonym: Pachymerus palliatus Costa, 1855.

Distribution in Libya: Cyrenica: Mancini, 1942a.

General Distribution: Austria, Bosnia, Bulgaria, Croatia, Czech Republic, Turkey, France, Hungry, Italy, Macedonia, Portugal, Romania, Russia, Slovakia, Slovenia, Spain, Algeria, Slovkia, Kaz-khstan, Switzerland, Ukraine, Egypt, Tunisia, Azerbaijan, Armenia, Syria, Georgia, Cyprus, Kirgizia, Tadzhikistan, Turkmenistan, Uzbekistan, and Libya.

Remarks: Species Brachyplax tenuis (Mulsant \& Rey, 1852) was listed as Brachyplax palliata (Costa, 1852) by Eckerlein \& Wagner, 1969.

\section{*Brachyplax tenuis tenuis (M \& R, 1852)}

Synonym: Brachyplax albidus Fieber, 1861.

Distribution in Libya: El Mej.

General Distribution: Cyprus, France, Greece, Hungary, Italy, Lesbos Isles, Portugal, Romania, Russia, Spain, Algeria, Egypt, Morocco, Caucasus, Turkestan, Turkey, and Libya.

\section{Genus: MetopoplaxFiebe, 180}

Type species: Pachymerus ditomoides Costa, 1847. 
*Metopoplax ditomoides (Costa, 1847)

Synonym: Pachymerus ditomoides Costa, 1847

Distribution in Libya: Benghazi.

General Distribution: France, Czech Republic, Hungary, Syria, Turkey, German, Holland, Malta, Portugal, Portugal, Spain, Switzerland, Tunisia, Morocco, Belgium, Bosnia, Bulgraia, Algeria, Morocco, Belgium, Bosnia, Bulgaria, Luxemburg, Macedonia, Malta, and Libya.

\section{SUBFAMILY: Atheneinae}

Genus: Artheneis Spinola, 1837

Type species: Artheneis foveolata Spinola, 1837.

\section{Artheneis alutacea Fieber, 1860}

Distribution in Libya: Fezzan: Rhoddua.

General Distribution: Macedonia, Algeria, Egypt, Greece, Italy, Tunisia, Cyprus, Jordan, Morocco, and Libya.

\section{Artheneis aegyptiaca Lindbg, 1939.}

Synonym Artheneis aegyptiaca Lindberg, 1939.

Distribution in Libya: Kufra Oasis, Zaraga, Bir Ygub (Mancini, 1940).

General Distribution: Yemen, Sudan, Saudi Arabia, Egypt, Syria, Iran, and Libya.

\section{*Artheneis balcanica (Kormilev, 1938)}

Synonym: Karamania balcanica Kormilev, N 1938.

Distribution in Libya: El Kufra.

General Distribution: Bulgaria, Greece, Ukraine, Azerbaijan, Turkmenistan, Iraq, Syria, Bulgaria, Tajikistan, Uzbekistan, Turkmenistan, Greece, Macedonia, Ukraine, Syria, Turkey, Cyprus, Uzbekistan, and Libya.

\section{*Artheneidea tenuicornis Kiritshenko, 1913}

Synonym: Artheneis chlorotica de Bergevin, 1930: Ann. Mus. Civ. Stor. Nat. Gen. 55: 35 Distribution in Libya: Jagbob (de Bergevin, 1930, Zavattri, 1934).

General Distribution: Libya, Iraq, Turkestan, Algeria, Egypt, Tunisia, Kazakhstan, China, Iran, Mongolia, Saudi Arabia, Uzbekistan, Tadzhikistan, Turkmenistan, Sudan.
Remarks: Artheneidea tenuicornis Kiritshenko, 1913 was listed as Artheneis chlorotica de Bergevin, 1930 by Eckerlein\& Wagner, 1969.

\section{Artheneis beieri Wagner, 1963.}

Artheneis beieri Wagner, 1963: Zootaxa 3408, pp. 1-33.

Distribution: $20 \mathrm{Km}$ west to Gasr Garabulli.

General distribution: Iran, Turkmenistan, Uzbekistan, Yemen, Sudan, Algeria, and Libya.

\section{REFERENCES}

Amyot, C., \& Serville, A. (1843). Histoire Naturelle des Insects Hemipteres Libraire Encyclopedique de Roret. Paris: Fain et Thunot, 1.

Ashlock, P. D. (1967). generic classification of the Orsillinae of the world (HemipteraHeteroptera: Lygaeidae).

China, W. E., \& Miller, N. C. E. (1959). Check-list and keys to the families and subfamilies of the HemipteraHeteroptera. British Museum (Natural History).

Cross, G. R., \& Scudder, G. (1963). The Australian Rhyparochromini (Hemiptera: Lygaeidae). Printed in Australia by WL Hawes, Govtt. Printer.

Eckerlein, H., \& Wagner, E. (1969). Die Heteropterenfauna Libyens. Acta Entomologica Musei Nationalis Pragae, 38, 155-194.

El-Meghrabi, M. (2001). External Morphology of adult Graphosoma semipunctatum (Fabricius),Family Pentatomidae, order Hemiptera-Heteroptera). Garyounis Scientific Journal, 14(No.1\&2), 117136.

El-Meghrabi, M. S. (1999). Field and Systematic studies on the family Pen-tatomidae (Order Heteroptera) from Egypt. Ph.D.Thesis, Faculty of science, Ento- 
mology department, Cairo University, 383.

El-Meghrabi, M. S. (2009). A preliminary survey of insect species at $\mathrm{El} \mathrm{Ku}$-fra and Jabel El Uweinat in Libya. Arab J.Pl.Prot, 27,Special Issue(Supplement)

El-Meghrabi, M. S. (2009). Survey of some Heteropterous insects in libya. Journal of Productivity and Development, 14(3), 747-758.

Faimaier, L. (1855). Notes sur quelques Hemipteres du Ma-roc. Rev.Ent.Caen, 351-355.

Gross, G. F. (1965). A revision of the Australian and New Guinea Drymini (Heteroptera-Lygaeidae). Records of the South Australian Museum, 15, 39-78.

Hoberlandt, L. (1953). Results of the Armstrong College Expedition to Siwa Oasis (Libyan Desert), 1935, under the leadership of Prof. J. Omer-Cooper. Hemiptera-Heteroptera. Bulletin de la Société Fouad Ier d'Entomologie, 37, 359-370.

Linnavuori, R. (1964). Hemiptera of Egypt, with remarks on some species of the adjacent Eremian region. Annales Zoologici Fennici,

Linnavuori, R. (1969). Contributions to the Hemipterous fauna of Egypt. Annales Entomologici Fennici,

Malipatil, M. B. (1978). Revision of the Myodochini (Hemiptera: Lygaeidae: Rhyparochrominae) of the Australian Region. Australian Journal of Zoology Supplementary Series, 26(56), 1-178.

Schuh, R. T., \& Slater, J. A. (1995). True bugs of the world (Hemiptera: Heteroptera): classification and natural history. Cornell UNIVERSITY press.

Scudder, G. (1962). The Ischnorhynchinae of the world (Hemiptera: Lygaeidae). Transactions of the Royal Entomological Society of London, 114(6), 163-194.

Slater, A. (1992). A genus level revision of western hemisphere Lygaeinae (Heteroptera: Lygaeidae) with keys to species. Revisión a nivel de género de Lygaeinae (Heteroptera: Lygaeidae) del hemisferio occidental con claves para las especies. The University of Kansas Science Bulletin., 55(1), 1-56.

Stål, C. (1864). Hemiptera africana (Vol. 1). ex officina Norstedtiana.

Stichel, W. (1961). Illustrierte Bestimmungstabellen der Wanzen II Europa (Heteroptera-Heteroptera Europae). BerlinHermsdorf, Berlin: Stichel, 169-907.

Vidal, J. (1949). Hémiptéres de L'Afrique Du Nord Et Des Pays Circummediterranéens: Travail Effectué Avec L'aide Du Service de la Défense Des Végétaux Du Maroc; Publié Avec Une Subventon de la Direction de L'Agriculture, Du Commerce Et Des Foréts. Institut Scientifique Chérifien.

Zavatari , E. (1934). Prodromo della Fauna della Libi. Co-operativa, VII, 123. 
نبذة مختصرة عن رتبة حشرات بق البذور Schilling, 1829 التابعة لمختلفة الأجنحة في ليبيا

$$
\begin{aligned}
& \text { مفتاح سليمان المغربي } \\
& \text { قسم علم الحيوان -كلية العلوم- جامعة بنغازي، بنغازي - ليييا }
\end{aligned}
$$

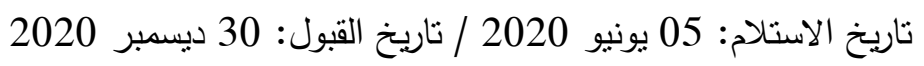

$$
\begin{aligned}
& \text { https://doi.org/10.54172/mjsc.v35i4.335:Doi }
\end{aligned}
$$

المستخلص: تتتاول الدراسة عرض شعب وأجناس وأنواع فصيلة حشرات بق البذور Lygaeidae و تمت دراستها مصحوبة

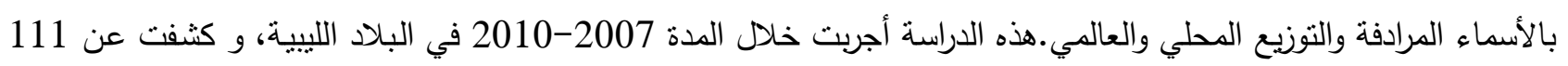
نوع موجود في ليييا، تحت 52 جنس تابع 8 تحت فصيلة. 31 نوع تسجل لأول مرة في ليبيا.

الكلمات المفتاحية: عائلة بق البذور Lygaeidae ، مختلفة الأجنحة، ليييا، المرادفات، توزيع محلي وعام، بق البذور . 